\title{
A New Methodology for Plutonium Homogeneity Level Determination in Sodium Fast Reactor Fresh Fuel From Uranium and Plutonium X-ray Mappings
}

\author{
L. Aufore ${ }^{1}$, J. Léchelle ${ }^{1}$ \\ ${ }^{1}$ CEA, DEN/DEC/SPUA F-13108 St-Paul-Lez-Durance, France
}

In the framework of the Fourth Generation program, the CEA studies a new concept of Sodium Fast Reactor (SFR) named ASTRID [1]. The reference fuel chosen for the reactor starting is uranium and plutonium mixed oxides. This type of fuel had been fabricated until 1999 during the PHENIX (French SFR prototype) operation. The industrial process led to homogeneous uranium /plutonium distributions in compliance with the fabrication specifications. Nowadays, specific R\&D programs deal with innovative processes in order to obtain $(\mathrm{UPu}) \mathrm{O}_{2}$ fuel with the same level of homogeneity [2]. In order to evaluate this feature, microstructural characterizations especially uranium and plutonium distributions are carried out using Electron Probe Micro Analysis (EPMA). Data post-treatments consist in measuring plutonium homogeneity level on a micrometer scale using initial mappings. This paper focuses on the development of a calculation tool using EPMA mappings which allows to qualify plutonium homogeneity level of industrial fuels, and to compare it to new R\&D fuels developed in the frame of ASTRID project.

Plutonium and uranium X-ray mappings are recorded using CAMECA SX 100 EPMA on sections of 4 oxide pellets fabricated in 1999 for PHENIX reactor. On each section, 3 fields randomly chosen of 1 $\mathrm{mm}^{2}$ (resolution 1024x1024 pixels) are collected. Measurements are taken on the $\mathrm{Pu}, \mathrm{U}$, and $\mathrm{O}$ peaks without subtracting the spectrum background. Uranium and plutonium X-ray mappings (in grey levels) are presented in figure 1. These images highlight a uniform grey level for $U$ and Pu with small zones in white color which are $\mathrm{U}$ or $\mathrm{Pu}$ enriched areas respectively and some small dark zones which can be either porosity or a lack of chemical element. $\mathrm{U}$ and $\mathrm{Pu}$ distributions (in grey levels) are reported on figure 2 and show that the 12 fields are very homogeneous on this scale and very reproducible for a pellet and for a pellet batch from a fabrication.

The methodology we developed uses at first, a preliminary basic image analysis processing especially to eliminate porosity, followed by mathematical calculations which are applied to plutonium X-ray mappings in grey levels. Based on the fact that homogeneity is reached as composition measurements on any sampled region yields to the same result, mathematical criteria as standard deviation relative to mean and median values, entropy and an autocorrelation factor related to the size of heterogeneity are well suited to describe this feature. Calculation of each criterion, at several resolution levels of the initial twelve images leads to a lot of information. Thus it was decided to treat the whole data set using a statistical data analysis method. The Principal Component Analysis (PCA) is a statistical procedure which uses an orthogonal transformation to convert a set of possibly correlated variables into a set of values of linearly uncorrelated variables called principal components [3]. Afterwards we determine a new graphical space with principal components as axes along which the data variance is maximum. On this graph, each point represents the level of homogeneity of each image relatively to the others. The axes $\mathrm{X}$ and $\mathrm{Y}$ are linked to a linear combination of every criteria and represent respectively $70 \%$ and $20 \%$ of the whole information. In the case of PHENIX pellets characterization Figure 3 shows results about the 4 industrial pellets. Compared to figure 2 we can appreciate on figure 3 very tiny differences between each pellet. This graphical $2 \mathrm{D}$ visualization allows to class pellets or samples in term of relative homogeneity, pellet 1 is the most homogeneous sample on figure 3 . With such a methodology we can 
also compare different fuels fabricated from different routes considering the set of images acquired on each sample. All the calculations are automatically realized using the software tool we specifically developed. This tool is still under test in order to validate the method on the basis of data from a larger panel of fuels. Moreover, this method could be widely applied to EPMA elemental mapping for materials comparison.

\section{References :}

[1] P. Le Coz et al, ICAPP 2013, Jeju island, Korea, 14-16 April 2013

[2] S. Vaudez et al, Journal of Nuclear Materials 442 (2013) 227-234

[3] M. Petrou, C. Petrou, "Image Processing: The Fundamentals", 2010 ed. John Wiley \& Sons
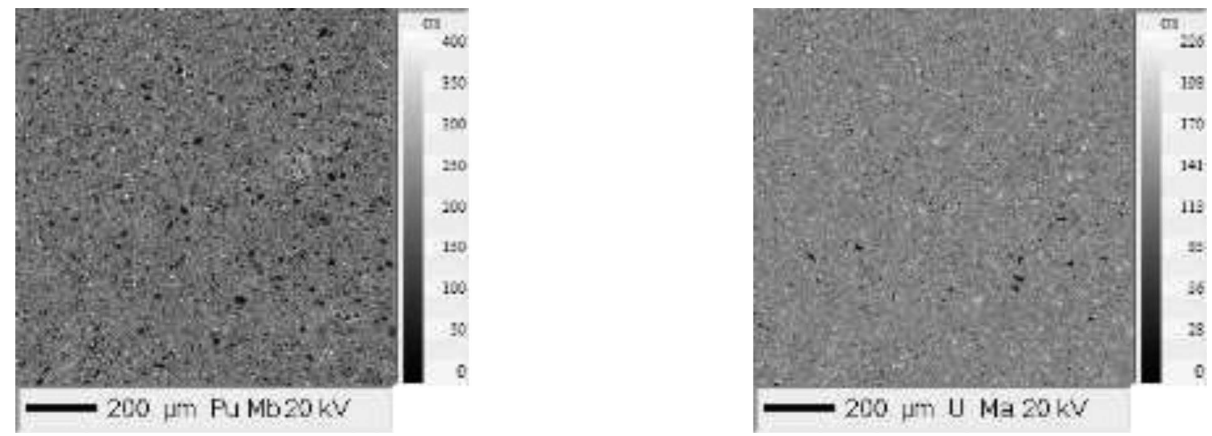

Figure 1. Plutonium and Uranium X-ray mappings measured on PHENIX pellets
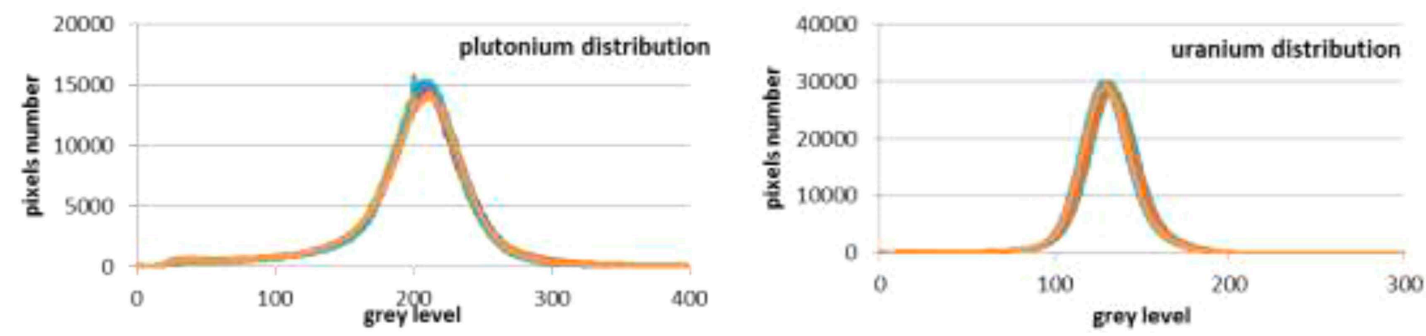

Figure 2. Grey level distributions for the twelve Plutonium and Uranium X-ray mappings acquired
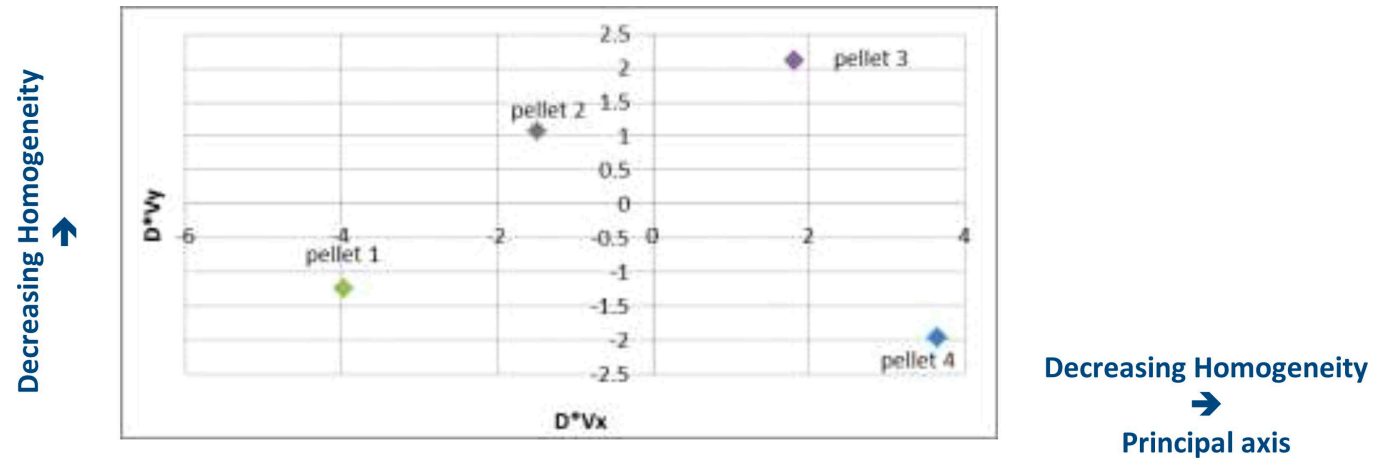

Figure 3. Graphical results of homogeneity level evaluation for 4 pellets of the industrial PHENIX fuel: pellet 1 is the most homogeneous sample for this set of data 